\title{
Development of high resolution thin film patterns on curved photocathode substrates of image tubes
}

\author{
L M RANGARAJAN and V R KATTI \\ Bhabha Atomic Research Centre, Trombay, Bombay 400085, India
}

\begin{abstract}
Fabrication of thin film patterns involves a sequence of photolithographic and chemical processes to define the high resolution features in thin films on the surface of the optical substrates. The substrates used in the present work were curved fused silica with radii of curvature $58 \mathrm{~mm}$ and $18 \mathrm{~mm}$ which are used as photocathode substrates for image converter and image intensifier tube devices. The resolution pattern and bar chart were successfully printed on these substrates using a secondary conformable photomask of Mylar.
\end{abstract}

Keywords. Photolithography; electron-optics; image tube; resolution; photoemission; integrated-circuits.

\section{Introduction}

The study of experimental electron optics of photoelectronic imaging devices either in practical electron valves or in demountable systems is possible only by using gold photocathode resolution patterns. An important aspect of the experimental electron optical demountable system (EEODS) is the photocathode and lamp combination, for on this depends the simplicity of the day-to-day operation. This requires a photocathode that can be exposed to air without being damaged. Metals, which are, ultravioletsensitive can satisfy this requirement. The required test pattern is defined in a mask on a quartz substrate and a photocathode evaporated on the top (Picat et al 1972). The mask is then illuminated from behind with an Uv lamp so that electrons are emitted from the clear areas of the mask and the pattern is converted into an electron image. The photoelectrons are then accelerated by a high electric field onto the phosphor screen (figure 1). Among the photocathode materials $\mathrm{Au}, \mathrm{Pd}$ and $\mathrm{CsI}$ are typical examples. The photoelectric work function of Au film is $4.492 \mathrm{eV}\left(\lambda_{\mathrm{th}}=2760 \AA\right)$. Hence uv excitation from an $\mathrm{Hg}$-lamp is sufficient to generate the required electron current density. Photolithographic contact printing method (IEEE 1975) was employed in this work for the replication of high resolution patterns from flat master masks to Mylar and curved quartz substrates.

\section{Experimental techniques}

The three important resolution test charts used as master masks for the entire study and analysis of electron optics of image devices were (a) RETMA pattern (b) Westinghouse bar chart (ET-1332A) (c) USAF 1951 resolving power target. The finest line of each pattern was $6 \mu \mathrm{m}, 6 \mu \mathrm{m}$ and $2 \mu \mathrm{m}$ width. Since the pattern cannot be directly transferred from the emulsion plate fiat master mask to the curved quartz substrates, a secondary 

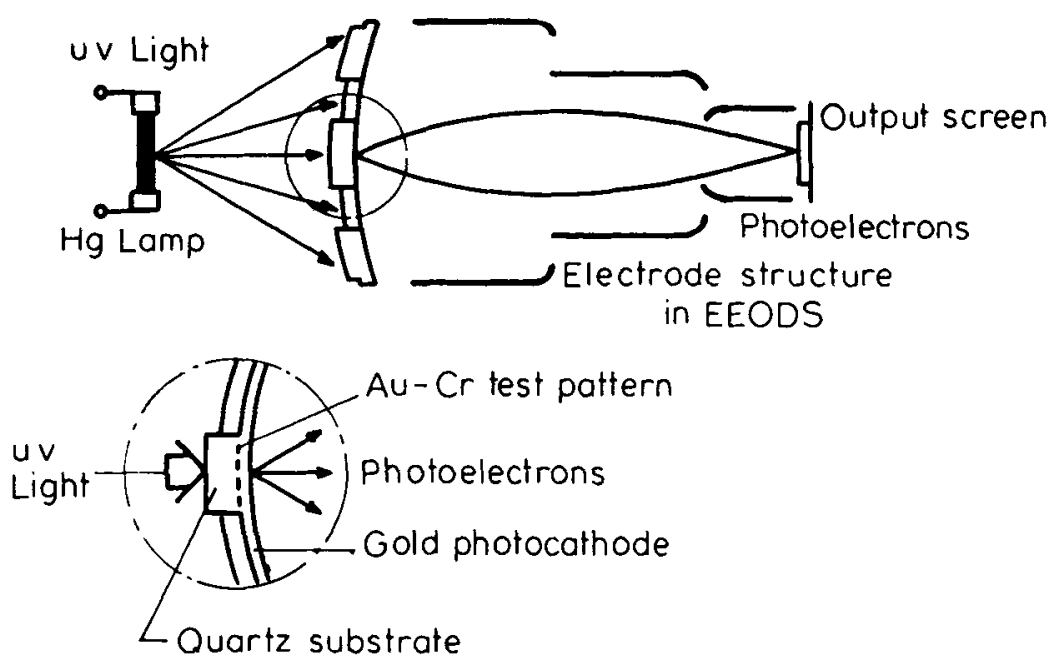

Figure 1. A gold photocathode resolution pattern inside a demountable electron-optical system.

conformable master mask was developed to be copied on the curved substrates, by contact printing. Of the many organic sheet materials, Mylar sheet has an attractive combination of properties such as mechanical strength, resistance to organic solvents, optical transparency and availability in various thicknesses with optical quality surfaces. The Mylar substrate is completely transparent so that optical realignment of masks is possible. The masks are durable and handling is easier than glass masks now used in photolithography. The dimension of Mylar does not change with temperature and humidity at ambient conditions. It was therefore selected as the substrate material for secondary mask. Photolithographic techniques involved deposition of metallic films on the given substrate followed by selective removal of portions of the film using organic solvents dispersed in photoresists which protect the film in the desired regions, while the unwanted and unprotected regions are etched away chemically. In general pattern delineation by optical lithography gives satisfactory resolution for features as small as $2 \mu \mathrm{m}$. Limit of line resolution is set by wavelength of uv light, $0.2 \mu \mathrm{m}$.

Deposition of $\mathrm{Cr}-\mathrm{Au}$ films as well as Ti-Pd-Au films on Mylar samples was independently carried out for getting satisfactory patterns. A Mylar sheet $(30 \times 20$ $\times 0.4 \mathrm{~mm}$ ) was washed with trichloroethylene and ultrasonically cleaned to remove fine dust particles and organic solvents on the surface, which are a potential source of pinholes. An aluminium strip placed below the Mylar (which buckles at $150^{\circ} \mathrm{C}$ ) served as a heat sink. Uniform layers of chromium $(100 \mathrm{~nm})$ and gold $(100 \mathrm{~nm})$ films were deposited in sequence on the Mylar substrate, using fast evaporation and employing double filament methods in a vacuum of $<10^{-5}$ torr. The filaments were of basket type made of tungsten. Chromium, in fact, sublimes above $1800^{\circ} \mathrm{C}$ and gold evaporates at $1000^{\circ} \mathrm{C}$. Shutter mechanism and double filaments were incorporated inside the vacuum evaporation plant.

A thin uniform layer $(0.3 \mu \mathrm{m})$ of Kodak negative photoresist or KTFR was coated on the AuCr layer by a photospinner. The centrifugal whirling of photoresists is the standard application technique for producing sub-micron thickness films of these materials. The viscosity of the resist solution and the speed of rotation of the whirler 
were optimized to obtain a thin, uniform, bubble and agglomeration-free photoresist coating on the Mylar substrate. For different samples tried with varying thickness of resist films, the resolution was found to vary inversely with resist thickness. It was prebaked at a temperature of $80^{\circ} \mathrm{C}$ for half an hour. Baking the photoresist layer prior to exposure is often helpful for this particular photofabrication process. The purpose of the prebake is to remove any residual photoresist solvent that has not been eliminated by air drying. Failure to prebake the layer of resist usually results in either degradation or complete failure of the image in the developer. The photoresist coated substrate was exposed for $7 \mathrm{sec}$, in a system which included a light source, provision for holding the substrate and mask in close contact and a microscope to observe the mask. The pattern was exposed with a high pressure $\mathrm{Hg}$ vapour lamp which produced nearly collimated light. Typical value of the exposure of light energy at the substrate was $10 \mathrm{~mW} / \mathrm{cm}^{2}$. Details of developing, postbaking and chemical etching were reported earlier (Sunita Singh et al 1980). A resolution of $6 \mu \mathrm{m}(80 \mathrm{lp} / \mathrm{mm})$ was observed on the secondary mask. To develop a conformable mask pattern of very high resolving power of $2 \mu \mathrm{m}(250 \mathrm{lp} / \mathrm{mm})$ like the one of USAF 1951 target, the experimental techniques were modified (Rangarajan 1982). Uniform layers of $\mathrm{Ti}(40 \mathrm{~nm})$ and $\mathrm{Pd} \cdot \mathrm{Au}(50 \mathrm{~nm})$ films were deposited sequentially on the Mylar sample in a pressure $<10^{-5}$ torr. Ti wire of 8 mil was wound on tungsten ribbon-stranded filament, for evaporation. Ti evaporates at $1800^{\circ} \mathrm{C}$. Pd-Au wire $(60 \% \mathrm{Pd}$ and $40 \%$ Au alloy composition) from basket type $\mathrm{W}$ filament evaporates at $900^{\circ} \mathrm{C}$. The photofabrication process was similar excepting for the following changes: the negative photoresist used in the present study was from Way-Coat (USA); the thickness of NPR coating was kept below $1 \mu \mathrm{m}$; line broadening due to light scattering under the dark portions of the mask was eliminated with welladjusted exposures and negligible mask-to-substrate separation as well as by using dark field and bright field patterns at appropriate times; chemical etching for Ti film was done from a solution of $70 \mathrm{cc} \mathrm{H}_{2} \mathrm{O}, 10 \mathrm{cc}$ of $48 \% \mathrm{Hf}$ and $20 \mathrm{cc}$ of $\mathrm{HNO}_{3}$ and the rate of etching was $18 \mu \mathrm{m}$ per min at $32^{\circ} \mathrm{C}$. The strong adhesive property of $\mathrm{Ti}$ on silica or Mylar substrates retained the fine details of the pattern after chemical etching. As many as 25 samples were prepared in the course of experiments. A resolution of $2 \mu \mathrm{m}$ was observed on the secondary mask which had very good contrast and high edge definition.

\section{Experimental results and discussion}

To evaluate the quality of the mask, the transmittance of different regions was measured with a micro-densitometer (type: Jarrel-ash Company) and a high precision recording microphotometer (model-23-500/501). Density of lines was 0 to 3.5 with a slit width of $1.4 \mu \mathrm{m}$. Consider a series of periodic bars and spaces such as a picket fence. The spatial dimension is the period. The spatial frequency, as with time frequency is just the inverse of this period i.e. $1 / T$ (figure 2). This is expressed in lines per millimeter or resolution as $\mathrm{lp} / \mathrm{mm}$. The contrast of the mask pattern is the difference in optical density of the opaque and transparent regions at that spatial frequency. The optical density, is defined as

$$
D=\log _{10}\left(I_{0} / I_{7}\right)
$$

where $I_{0}$ and $I_{l}$ are intensity of incident and transmitted light. In figure 2, 1 is the black 
or grey bar and 2 is the white or full transmission bar,

$$
\text { contrast }=C=D_{1}-D_{2}=\log _{10}\left(I_{T_{2}} / I_{T_{1}}\right) \text {. }
$$

This contrast value was determined at different resolution values, $R$. While expressing the results of $C=f(R)$, values were normalized. A thumb rule is that the slit width should be at least one-third of the resolving power. The output of the microdensitometer was plotted on a recorder and looked like a square wave response. The method is analogous to obtaining the pulse response of an amplifier. The contrast of the pattern details described on the Mylar samples and the reference photographic emulsion mask is shown in figure 3.

The curved quartz substrates were coated with $\mathrm{Cr}$ and $\mathrm{Au}$ films in sequence and a thin layer $(0.5 \mu \mathrm{m})$ of positive photoresist spun on them. The Mylar pattern was transferred on the silica glass substrates as a contact print by making the Mylar sheet take the shape of curved substrates of $60 \mathrm{~mm}$ and $18 \mathrm{~mm}$ radii of curvature in the two cases. This is possible by using a specially designed fixture (figure 4). Close contact between the Mylar mask and quartz substrate was achieved by applying mechanical pressure. While the top and bottom metallic flanges are tightened (with bolts and nuts), the teflon

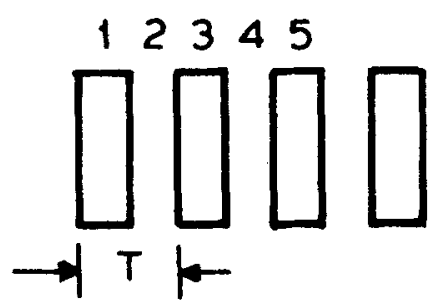

Figure 2. Spatial frequency.

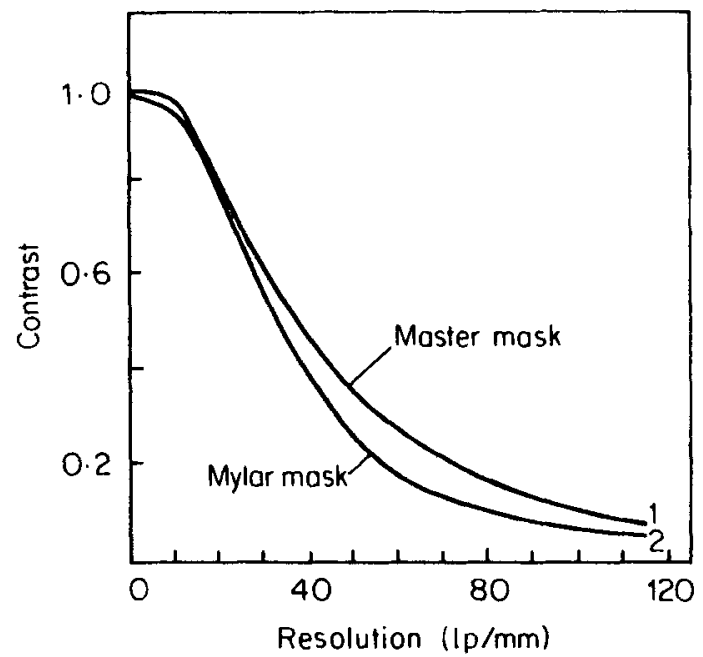

Figure 3. Contrast curves. 


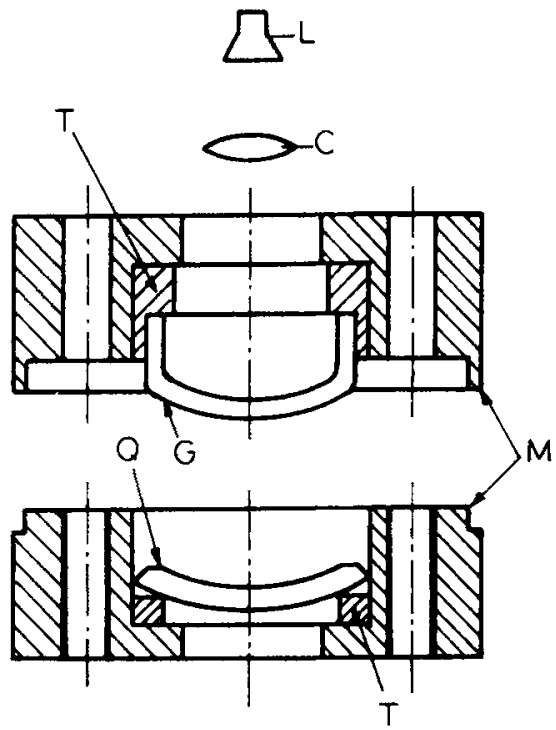

Figure 4. Mechanical and optical set-up for photomasking (L-Lamp; C-Collimating lens; T-Teflon runners; M-Special fixture; G-Glass plate; Q-Curved quartz plate $(\mathrm{RC}=18 \mathrm{~mm})$ ).

runners take care of the extra-pressure and guide the glass plate and quartz plate to a proper alignment. Here again, the usual photolithographic techniques were employed for printing the resolution pattern on the quartz substrate. While transferring the details to cathode substrates, special precaution has to be exercised to completely eliminate the line width variations in photoresist patterns on profiled surfaces. This has been made possible by using (i) mask alignment apparatus with new techniques such as optical contact or coupling liquid (DoW Corning Q2-3067, Refractive index is of quartz, FoP coupling of image tubes) between the Mylar pattern and the resist surface and (ii) by using dark field and bright field patterns appropriately with PPR or NPR during printing.

After transferring the pattern on the curved substrate, a thin film of gold of thickness $25 \mathrm{~nm}$ was evaporated over the pattern in a vacuum of $10^{-6}$ torr to form a semitransparent gold photocathode. The curved gold photocathode and the electrodes of an image tube were mounted and aligned on an electron-optical bench contained in an evacuated chamber, whose dynamic vacuum was $10^{-5}$ torr. A Hanovia uv lamp ( $500 \mathrm{~W}$ ) was used for exciting the Au photocathode. Photocurrents of the order of $10^{-7}$ to $10^{-8} \mathrm{~A} \mathrm{~cm}^{-2}$ could be obtained from the photocathode. The electron optical image was reconverted into visible image at the screen by cathodoluminescence, which could be viewed externally by a naked eye or by an optical microscope when necessary. The limiting resolution of the tube in the position of best focus was read on the screen. By measuring the widths of the pattern on the image at centre and at known distances from the centre, the magnification and distortion characteristics were obtained. For a typical tube with a radius of curvature of gold photocathode substrate as $18 \mathrm{~mm}$, the paraxial resolution obtained was $50 \mathrm{lp} / \mathrm{mm}$ and the magnification is 0.978 . 


\section{Conclusions}

While employing conventional photolithographic methods, new techniques have been introduced in the preparation of a conformable Mylar mask with resolution details of $2 \mu \mathrm{m}$ and, in the final process of printing such test patterns on curved quartz substrates. Such electronic patterns contribute immensely to the experimental electron optics studies of image tubes, and analysis of tube characteristics such as image details, aberrations etc which give image tube performance of practical importance. An important aspect of EEODS and allied techniques is that it forms the basic requirements of electron image projection systems for making master masks of $1 \mu \mathrm{m}$ details or smaller features required in integrated circuit technology. This is a fast growing field utilizing the principles of electron optics of photoelectronic imaging devices and electron-resist technology.

\section{Acknowledgements}

The authors thank Professor K V Ramanathan, Head, Solid-State Electronics Group, Tata Institute of Fundamental Research for allowing use of facilities and for guidance during the course of this work.

\section{References}

IEEE 1975 Pattern generation and microlithography, IEEE Trans Special issue ED-22, 369

Picat J P, Cambes M, Felenbok P and Fort B 1972 The electron Camera used in a reflective mode; Adv. Electron. Electron Phys. A33 557

Rangarajan L M 1982 A study of electron-optics in photoelectronic imaging devices, Ph.D. Thesis, Bombay University, Bombay

Sunita Singh, Rangarajan L M and Pishorady T U 1980 Application of photolithography to development of photocathodes for electron optical imaging devices; J. IETE 26190 